\title{
Two-dimensional electronic spectroscopy signatures of the glass transition
}

\author{
K.L.M. Lewis, J.A. Myers, F. Fuller, P.F. Tekavec and J.P. Ogilvie* \\ Department of Physics and Biophysics, University of Michigan, Ann Arbor, MI, USA
}

\begin{abstract}
Two-dimensional electronic spectroscopy is a sensitive probe of solvation dynamics. Using a pump-probe geometry with a pulse shaper [Optics Express 15 (2007), 16681-16689; Optics Express 16 (2008), 17420-17428], we present temperature dependent 2D spectra of laser dyes dissolved in glass-forming solvents. At low waiting times, the system has not yet relaxed, resulting in a spectrum that is elongated along the diagonal. At longer times, the system loses its memory of the initial excitation frequency, and the 2D spectrum rounds out. As the temperature is lowered, the time scale of this relaxation grows, and the elongation persists for longer waiting times. This can be measured in the ratio of the diagonal width to the anti-diagonal width; the behavior of this ratio is representative of the frequency-frequency correlation function [Optics Letters 31 (2006), 3354-3356]. Near the glass transition temperature, the relaxation behavior changes. Understanding this change is important for interpreting temperature-dependent dynamics of biological systems.
\end{abstract}

Keywords: Multidimensional spectroscopy, two-dimensional electronic spectroscopy, glass transition

\section{Introduction}

Two-dimensional electronic spectroscopy (2DES) is a useful tool for studying the dynamics of complex biological systems. Its ability to resolve ultrafast dynamics has been shown to be particularly useful in photosynthetic systems, which contain many coupled chromophores that absorb in similar wavelength ranges [2]. Low-temperature 2D studies of these systems can give additional insight in resolving individual excitations and couplings where broad, room-temperature peaks overlap. When interpreting 2D spectra at different temperatures, however, it is important to understand the role of solvent-solute interactions, and how these change with temperature. At low temperatures, many commonly used solvents for biological samples will undergo a phase transition to a glassy state, and this transition can have a large effect on solvation dynamics and spectral diffusion.

2DES has been shown to be a good probe of solvent relaxation dynamics [5]. This study uses a reporter dye molecule to study the changes in the local solvent environment in a glass-forming liquid as a function of temperature. When a dye is dissolved in a solvent, the local solvent environment modifies the dye's electronic structure. Differences in this environment lead to a range of absorption frequencies and an inhomogeneously broadened line shape. Spectral diffusion, or the sampling of different solvent configurations, is manifested by a broadening of the $2 \mathrm{D}$ line shape along the anti-diagonal for long waiting time delays $[5,10]$. In a 2D spectrum, the ellipticity of the line shape has been shown to closely

\footnotetext{
${ }^{*}$ Corresponding author: J.P. Ogilvie, Department of Physics and Biophysics, University of Michigan, 450 Church Street, Ann Arbor, MI 48109, USA. Tel.: +1 734615 0485; Fax: +1 734764 5153; E-mail: jogilvie@ umich.edu.
} 
follow the frequency-frequency correlation function that reflects the fluctuating solvent environment of the reporter dye [6].

Other spectroscopic techniques can also be used to probe solute behavior near the glass transition of the solvent. EPR, for instance, can recover information about the rotational correlation time as a function of temperature [11]. Single molecule fluorescence lifetime and linear dichroism studies can identify different polymer conformers. The preferred relaxation processes of the polymer change with temperature, particularly near the glass transition [1].

Above the glass transition temperature, reducing temperature slows the rate of spectral diffusion, since it reduces the speed of solvent reorganization. At temperatures below the glass transition, the system exhibits characteristically slower spectral diffusion caused by the "freezing out" of solvent motions. Thus the phase transition will be visible by a change in behavior of the frequency-frequency correlation function at the transition temperature. In this work we show experimental data that illustrate the changing ellipticity of the 2D line shape as a function of temperature, as well as the difference in solvent relaxation rates at room temperature and at $77 \mathrm{~K}$. The differences of the 2D line shape observed above and below the glass transition temperature $\left(T_{\mathrm{g}}\right)$ illustrate the method's ability to resolve solvent dynamics impacting the dye's electronic structure.

\section{Experimental methods}

A titanium sapphire oscillator (Synergy, Femtolasers) seeds a regenerative amplifier (Spitfire Pro, Spectra Physics) and produces $800 \mathrm{~nm}, 1 \mathrm{~mJ}$ pulses with a repetition rate of $1 \mathrm{kHz}$. This beam is split and fed into two independently tunable non-collinear optical parametric amplifiers (NOPAs). We perform 2DES in the "pump-probe" geometry [3]. The first NOPA creates a pump beam which is sent into an acousto-optic pulse shaper (Dazzler, Fastlite) in order to create a collinear pair of nearly transformlimited pump pulses of $12 \mathrm{~nJ}$ with a variable time delay, $t_{1}$. This delay is scanned and Fourier transformed to create the $v_{1}$ axis of the 2D spectra. The output of the second NOPA is compressed with a prism compressor and acts as a probe with an energy of $7 \mathrm{~nJ}$. The probe beam is crossed at a small angle with respect to the pump beam. Upon excitation by the pump pulses, the probe pulse interacts with the sample after a "waiting time" delay $t_{2}$ during which the system is allowed to relax. The 2D signal is emitted collinearly with the probe beam, and is spectrally resolved at $1 \mathrm{kHz}$ in a Horiba Jobin Yvon iHR320 spectrometer and Pixis 100B CCD camera, providing the $v_{3}$ axis of the 2D spectra. This setup is described in detail in [8].

In this study we used cresyl violet acetate (Fluka) and Nile Blue A (Sigma) as the reporter dyes, dissolved in a 1:1 (v/v) mixture of ethanol and propylene glycol $\left(T_{\mathrm{g}}=150 \mathrm{~K}[6]\right)$. The samples were held in a $250 \mu \mathrm{m}$ thick sample cell, with dye concentrations adjusted to yield an optical density of 0.25 at the absorption maxima. The samples were temperature controlled within a liquid nitrogen cryostat (MicrostatN, Oxford Instruments).

\section{Experimental results}

Two-dimensional electronic spectroscopy can serve as a probe of the liquid-glass phase transition through a number of different measures. The first method uses a temperature series of $2 \mathrm{D}$ spectra at a 

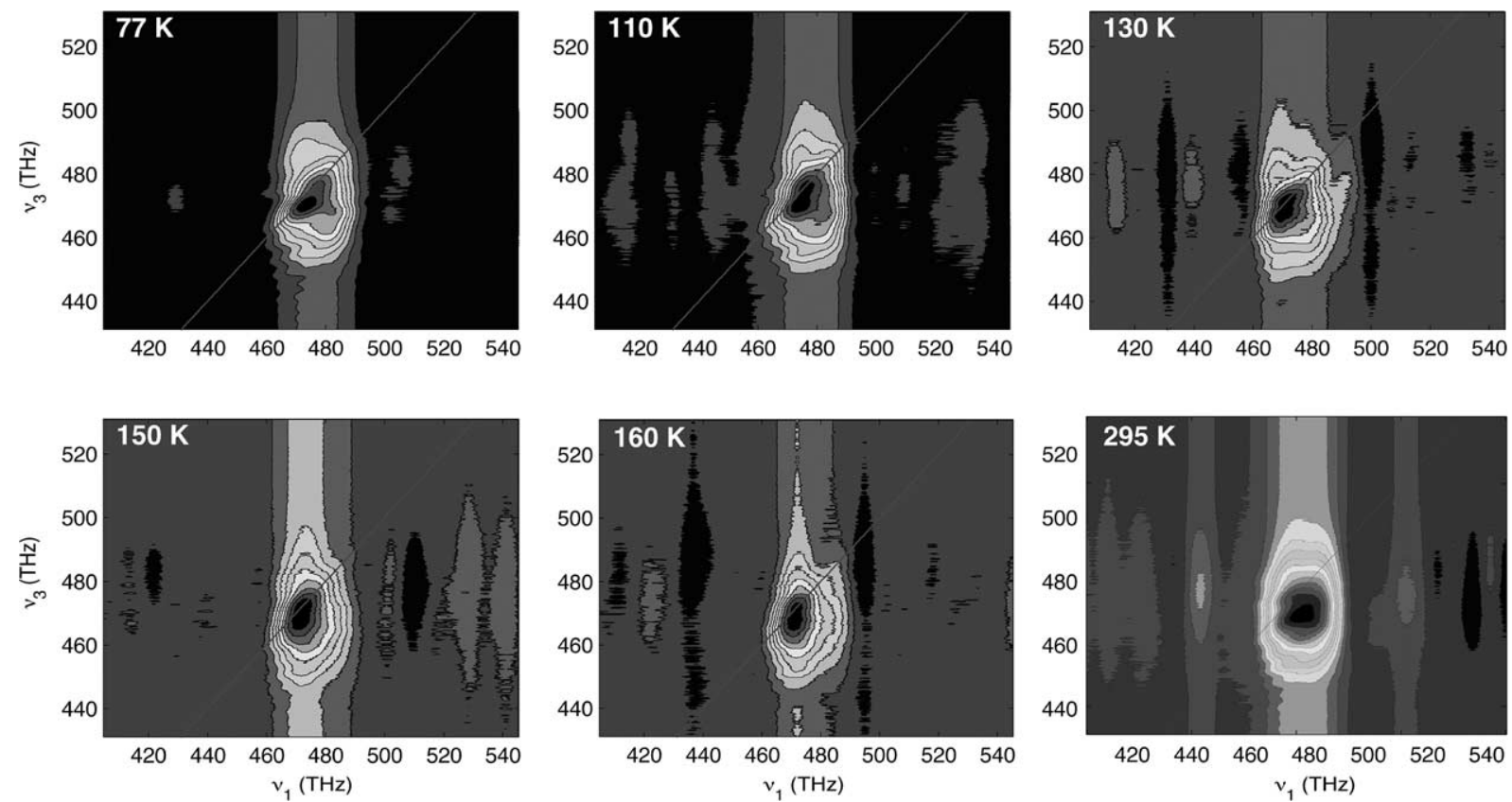

Fig. 1. Absorptive spectra of Nile Blue dissolved in a 1:1 (v/v) mixture of ethanol and 1,2-propanediol for a waiting time of $t_{2}=300 \mathrm{fs}$ at different temperatures above and below the glass transition. Both the pump and probe beams are centered at $630 \mathrm{~nm}$.

constant waiting time $t_{2}$ and examines the ellipticity of the 2D peak shape as the temperature changes [6]. As the temperature is lowered, the ellipticity (and hence correlation) for a given waiting time increases as solvent relaxation modes are frozen out.

Figure 1 shows a temperature series of 2D spectra for Nile Blue A in a mixture of ethanol and propylene glycol at a constant waiting time $t_{2}=300 \mathrm{fs}$. The diagonal peak shape is visually more elliptical at lower temperatures, indicating a higher degree of correlation. Nile Blue contains a $590 \mathrm{~cm}^{-1}$ vibrational mode that is apparent in this data as a secondary diagonal peak and a cross-peak at low temperatures [9]. At room temperature, these individual peaks are unresolved due to thermal broadening. This structure complicates a quantitative measurement of the ellipticity; the diagonal and anti-diagonal widths of an individual peak can appear broader than they are due to contributions from the neighboring peaks. Nevertheless, the higher ellipticity at lower temperatures is consistent with the expected behavior of slower spectral diffusion below the glass transition temperature.

We also studied the rate of solvent relaxation as a function of temperature by recording $2 \mathrm{D}$ spectra at different waiting times and comparing their ellipticities. At high temperatures, the solvent reorganizes quickly, reflecting a rapid decay of the frequency-frequency correlation function. This decay is significantly slowed in a low temperature, glassy state. Figure 2 shows a $t_{2}$ series of cresyl violet acetate dissolved in a mixture of ethanol and propylene glycol at both room temperature and $77 \mathrm{~K}$. At room temperature the line shape broadens rapidly, while the equivalent low temperature scans show little variation on the same time scale. The persistence of the elongation in the spectra indicates that many of the solvent relaxation pathways are frozen out below the glass transition. 
(a)

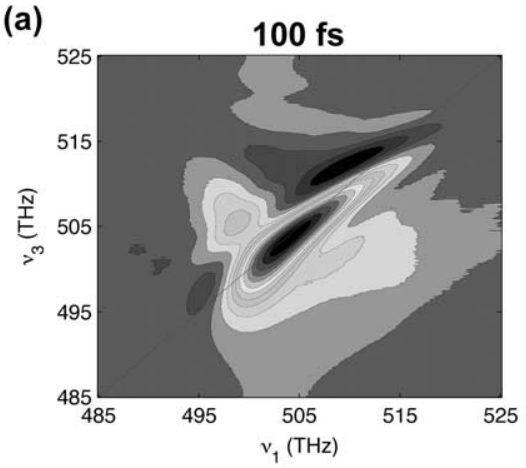

(d)

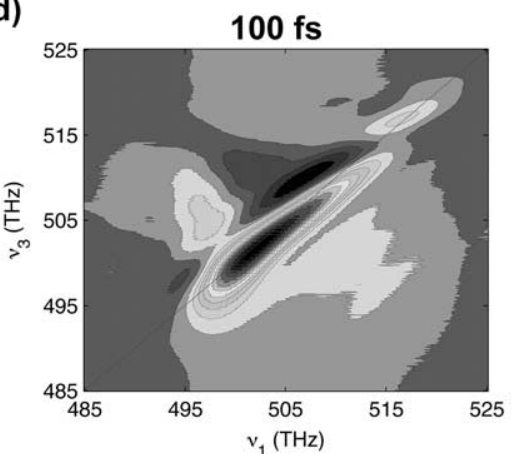

(b)

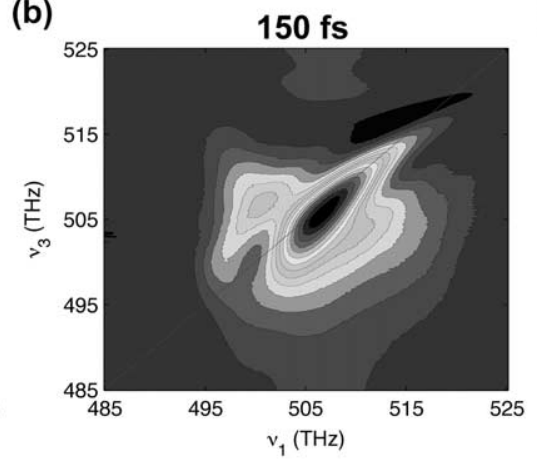

(e)

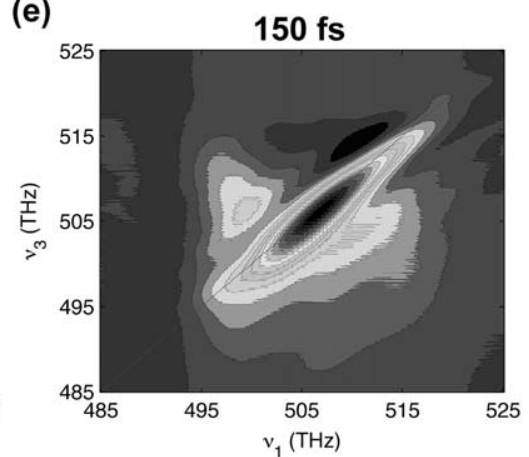

(c)

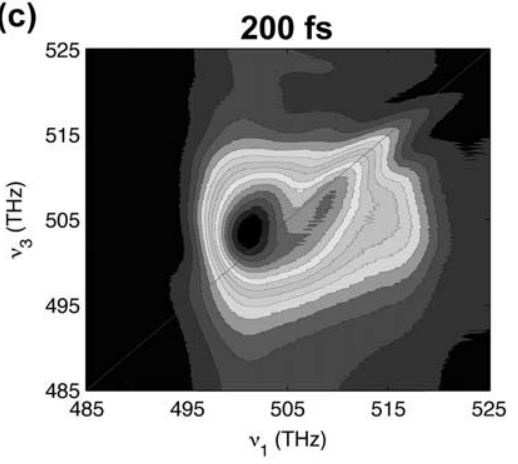

(f)

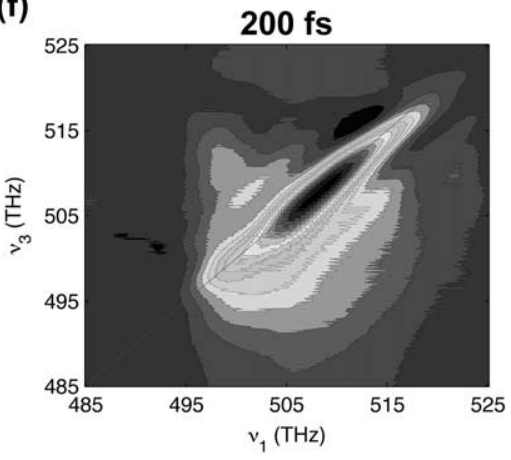

Fig. 2. Absorptive spectra of cresyl violet acetate dissolved in a 1:1 (v/v) mixture of ethanol and propylene glycol; (a)-(c) are at room temperature and (d)-(f) are at $77 \mathrm{~K}$. From left to right the $t_{2}$ values are 100, 150 and $200 \mathrm{fs}$. The pump and probe pulses are centered at $590 \mathrm{~nm}$.

\section{Discussion and summary}

In this paper we have shown experimental data demonstrating the temperature dependent characteristics of 2D line shapes that reflect the solvent relaxation dynamics of reporter dyes in glass-forming liquids. For low values of $t_{2}$, the 2D spectra are elongated along the diagonal, indicating that the system is inhomogeneously broadened. As $t_{2}$ increases, the molecules lose memory of their initial excitation frequency and the peak rounds. As the solution is cooled, this relaxation takes longer due to slower solvent reorganization. In a simple system, ellipticity is a good measure of the system-bath correlation function $[6,7]$ :

$$
M(t)=\frac{\langle\delta \omega(0) \cdot \delta \omega(t)\rangle}{\left\langle\delta \omega^{2}(t)\right\rangle}=\frac{a^{2}-b^{2}}{a^{2}+b^{2}} .
$$

Here, $a$ is the width of the peak along the diagonal and $b$ is the anti-diagonal width, while $\delta \omega(t)$ is represents the time fluctuations of the molecule's electronic transition due to interactions with the solvent.

By studying the ellipticity of the peak as a function of temperature and $t_{2}$, we see evidence of the glass transition, manifested by significantly slower spectral diffusion. The ellipticity measurement gives direct insight into the correlation function, allowing us to monitor the time constants of correlation loss as a function of temperature. Our data contains vibronic structure, which adds complexity to the 2D line 
shape, making the peak ellipticity somewhat ill-defined. Future work in this area will account for this structure. Beyond observing the glass transition, this work is a stepping stone towards exploring fast environmental changes of optical chromophores embedded in proteins.

\section{Acknowledgements}

The authors gratefully acknowledge the support of the Office of Basic Energy Sciences of the US Department of Energy (contract FG02-08ER15904) and the National Science Foundation.

\section{References}

[1] E. Braeken, G. De Cremer, P. Marsal, G. Pèpe, K. Müllen and R.A.L. Vallée, Single molecule probing of the local segmental relaxation dynamics in polymer above the glass transition temperature, Journal of the American Chemical Society 131 (2009), 12201-12210.

[2] T. Brixner, J. Stenger, H.M. Vaswani, M. Cho, R.E. Blankenship and G.R. Fleming, Two-dimensional spectroscopy of electronic couplings in photosynthesis, Nature 434 (2005), 625-628.

[3] S.M.G. Faeder and D.M. Jonas, Two-dimensional electronic correlation and relaxation spectra: theory and model calculations, Journal of Physical Chemistry A 103 (1999), 10489-10505.

[4] E.M. Grumstrup, S.-H. Shim, M.A. Montgomery, N.H. Damrauer and M.T. Zanni, Facile collection of two-dimensional electronic spectra using femtosecond pulse-shaping technology, Optics Express 15 (2007), 16681-16689.

[5] J.D. Hybl, A. Yu, D.A. Farrow and D.M. Jonas, Polar solvation dynamics in the femtosecond evolution of two-dimensional Fourier transform spectra, Journal of Physical Chemistry A 106 (2002), 7651-7654.

[6] K. Lazonder, M.S. Pshenichnikov and D.A. Wiersma, Easy interpretation of optical two-dimensional correlation spectra, Optics Letters 31 (2006), 3354-3356.

[7] K. Lazonder, M.S. Pshenichnikov and D.A. Wiersma, Echo-peak shift fails to resolve the liquid-glass phase transition, Chemical Physics Letters 449 (2007), 255-259.

[8] J.A. Myers, K.L.M. Lewis, P.F. Tekavec and J.P. Ogilvie, Two-color two-dimensional Fourier transform electronic spectroscopy with a pulse-shaper, Optics Express 16 (2008), 17420-17428.

[9] K. Ohta, D.S. Larsen, M. Yang and G.R. Fleming, Influence of intramolecular vibrations in third-order, time-domain resonant spectroscopies. II. Numerical calculations, Journal of Chemical Physics 114 (2001), 8020-8039.

[10] S.T. Roberts, J.J. Loparo and A. Tokmakoff, Characterization of spectral diffusion from two-dimensional line shapes, Journal of Chemical Physics 125 (2006), 084502.

[11] M.F. Santangelo, M. Levantino, A. Cupane and G. Jeschke, Solvation of a probe molecule by fluid supercooled water in a hydrogel at $200 \mathrm{~K}$, Journal of Physical Chemistry B 112 (2008), 15546-15553. 


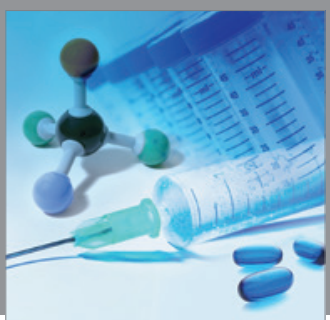

International Journal of

Medicinal Chemistry

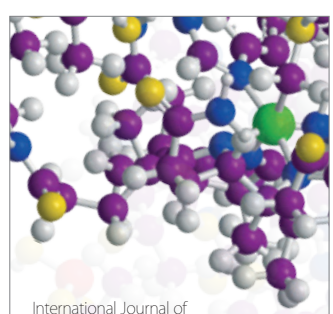

Carbohydrate Chemistry

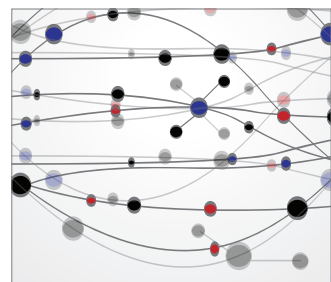

The Scientific World Journal
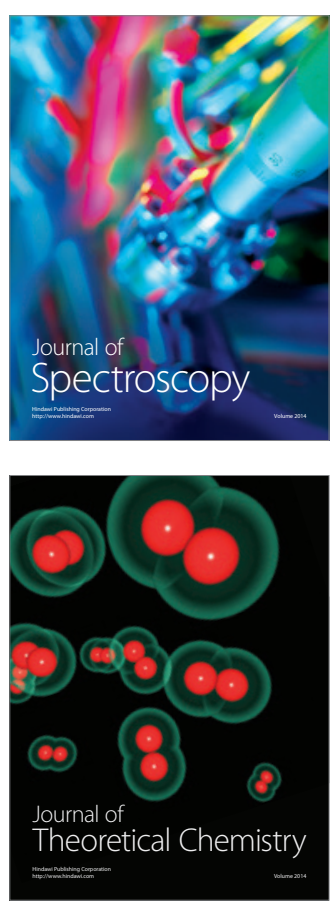
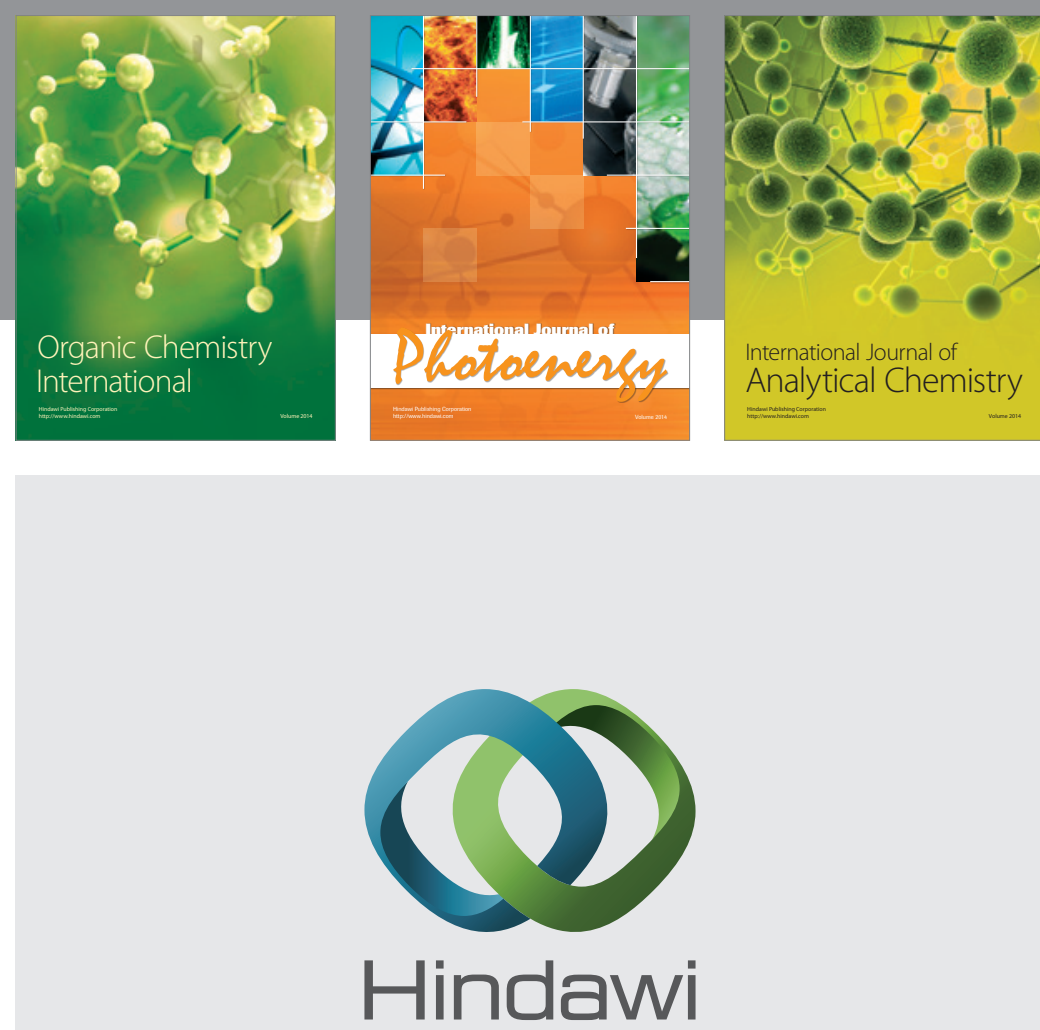

Submit your manuscripts at

http://www.hindawi.com
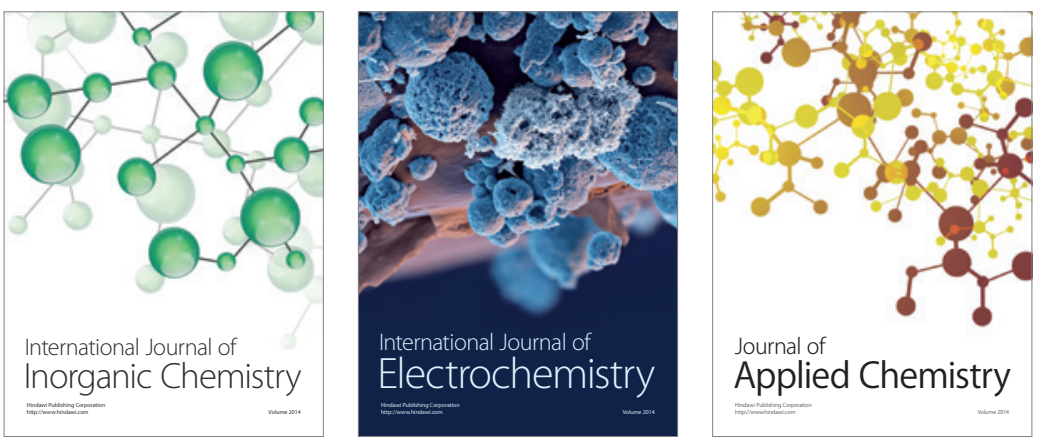

Journal of

Applied Chemistry
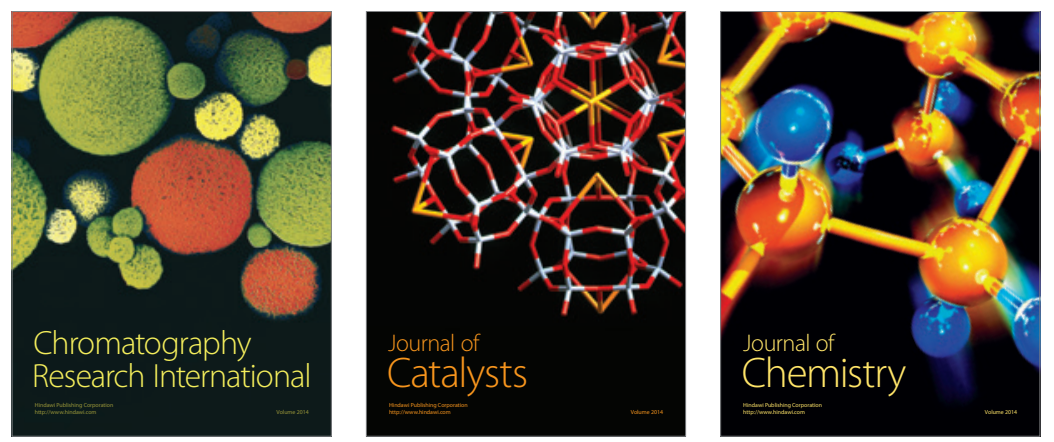
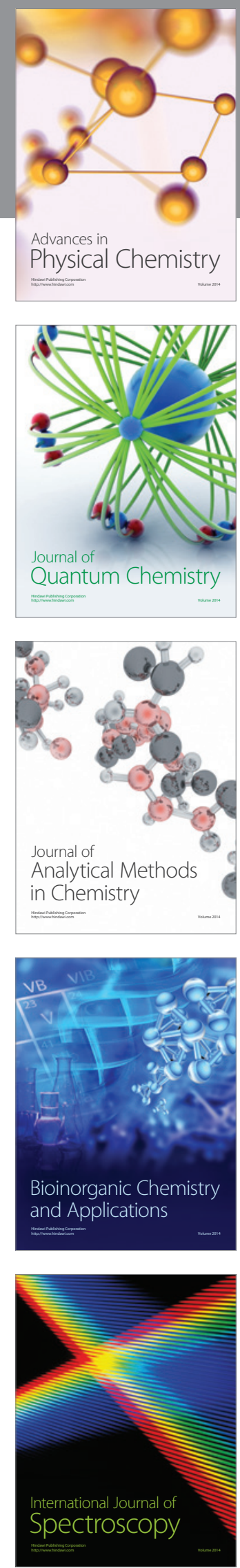